\title{
LncRNA SNORD3A specifically sensitizes breast cancer cells to 5 -FU by sponging miR-185-5p to enhance UMPS expression
}

\author{
Liyun Luo', Jianlei Zhang ${ }^{1}$, Hailin Tang $\mathbb{D}^{2}$, Dongfeng Zhai ${ }^{1}$, Danqing Huang ${ }^{1}$, Li Ling ${ }^{1}$, Xiaorong Wang ${ }^{1}$, Ting Liu', \\ Qiong Zhang ${ }^{1}$, Zhijie Zhang ${ }^{1}$, Zhimin $\mathrm{He}^{1}$ and Guopei Zheng ${ }^{1}$
}

\begin{abstract}
Breast cancer is the most common cancer type in women. Long non-coding RNAs (IncRNAs) have been reported as potential new diagnostic markers, prognostic factors, and therapeutic targets in cancer. However, the specific roles and mechanisms of IncRNAs in breast cancer remain to be elucidated. Here we demonstrated the downregulation of InCRNA SNORD3A in breast cancer cells and tissues and verified its non-protein-coding property. SNORD3A overexpression had no effect on cell proliferation but specifically sensitized breast cancer cells to 5-fluorouracil (5-FU) in vitro and in vivo. Mechanistically, SNORD3A exerts its effect via enhancing uridine monophosphate synthetase (UMPS) protein expression. SNORD3A acts as a competing endogenous RNA for miR-185-5p, leading to UMPS protein upregulation. miR-185-5p overexpression disrupted the effect of SNORD3A on chemosensitization to 5-FU in vitro and in vivo. Moreover, Meis1 overexpression transcriptionally promotes SNORD3A expression, and Meis1 is downregulated in breast cancer cells and tissues. In breast cancer tissues, SNORD3A level positively correlates with Meis 1 and UMPS protein levels, whereas miR-185-5p level negatively correlates with UMPS protein level. High SNORD3A transcript and Meis1 and UMPS protein levels predicts a better outcome, but high miR-185-5p level predicts a worse outcome in breast cancer patients receiving 5-FU-based chemotherapy. Our findings indicate that Meis1-regulated SNORD3A specifically sensitizes breast cancer cells to 5-FU via enhancing UMPS expression. The SNORD3A-UMPS axis may serve as a potential biomarker and therapeutic target to improve the efficacy of 5-FU-based chemotherapy for breast cancer patients.
\end{abstract}

\section{Introduction}

Breast cancer is the most common cancer type in women, accounting for approximately $25 \%$ of new cancer

\footnotetext{
Correspondence: Zhijie Zhang (zjiezhang@126.com) or Zhimin He (hezhimin2005@yahoo.com) or Guopei Zheng (zhengguopei@126.com) ${ }^{1}$ Affiliated Cancer Hospital \& Institute of Guangzhou Medical University; Guangzhou Municipal and Guangdong Provincial Key Laboratory of Protein Modification and Degradation; The State Key Laboratory of Respiratory; Guangzhou Key Laboratory of "Translational Medicine on Malignant Tumor Treatment", Hengzhigang Road 78\#, 510095 Guangzhou, Guangdong, China ${ }^{2}$ Department of Breast Oncology, State Key Laboratory of Oncology in South China, Sun Yat-sen University Cancer Center, Collaborative Innovation Center for Cancer Medicine, Dongfeng Road 651 E, 510060 Guangzhou, Guangdong, China

These authors contributed equally: Liyun Luo, Jianlei Zhang, Hailin Tang Edited by E. Candi
}

cases $^{1}$. Despite great advances in diagnosis and treatment strategies over the past decades, approximately $15 \%$ of the cancer-related deaths in women are due to breast cancer ${ }^{2}$. Breast cancer is a heterogeneous cancer type that exhibits a variety of histopathological characteristics and genetic alterations, with varying clinical outcomes. Based on intrinsic gene expression profiling, breast cancer can be divided into five major subtypes: luminal A, luminal B, HER2-positive breast cancer, triple-negative breast cancer (TN-BC), and normal breast-like subtype. TN-BC includes the basal-like and claudin-low subtypes ${ }^{3-5}$. The therapeutic strategy depends on the subtype and can include endocrine therapy, anti-HER2 targeting therapy, and chemotherapy. However, chemotherapy resistance

\section{(c) The Author(s) 2020}

(c) (i) Open Access This article is licensed under a Creative Commons Attribution 4.0 International License, which permits use, sharing, adaptation, distribution and reproduction cc in any medium or format, as long as you give appropriate credit to the original author(s) and the source, provide a link to the Creative Commons license, and indicate if changes were made. The images or other third party material in this article are included in the article's Creative Commons license, unless indicated otherwise in a credit line to the material. If material is not included in the article's Creative Commons license and your intended use is not permitted by statutory regulation or exceeds the permitted use, you will need to obtain permission directly from the copyright holder. To view a copy of this license, visit http://creativecommons.org/licenses/by/4.0/. 
remains a major obstacle for successful cancer treatment of all breast cancer types, resulting in recurrence, metastasis, and poor outcome ${ }^{6}$. Therefore, identifying strategies to overcome chemoresistance and increase chemosensitivity in breast cancer is critical to improve treatment efficacy.

Emerging evidence has revealed long non-coding RNAs (lncRNAs) as major regulators in both physiological and pathophysiological processes. LncRNAs are endogenous transcripts that are $>200$ nucleotides (nt) and lack protein-coding potential ${ }^{7}$. LncRNAs have been reported to be involved in a variety of biological activities, including proliferation, differentiation, migration, invasion, cell cycle, stem cell pluripotency, and lineage differentiation as well as cancer development and progression ${ }^{8-10}$. LncRNAs exert their roles at multiple mechanistic levels, such as regulating gene transcription, mRNA stability and translation, protein abundance and location, and chromatin and protein conformation ${ }^{11,12}$. Recent studies have revealed lncRNAs deregulation in a variety of cancer types and showed that lncRNAs can exhibit oncogenic function, cancer-suppressor function, or both ${ }^{13-16}$. Several lncRNAs have been shown to be involved in breast cancer. LncRNA NKILA binds nuclear factor (NF)-kB/inhibitor of $\mathrm{kB}(\mathrm{IkB})$ and masks the phosphorylation motif of $\mathrm{IkB}$ to inhibit IkB kinase-induced IkB phosphorylation and NF$\mathrm{kB}$ activation, thus preventing breast cancer metastasis ${ }^{17}$. We previously reported that the IncRNA LINC01638 was highly expressed in the TN-BC subtype and LINC01638 activates MTDH-Twist1 signaling by preventing SPOPmediated c-Myc degradation to maintain the epithelial-mesenchymal transition and cancer stem celllike state of TN-BC cells ${ }^{12}$. However, the specific roles and mechanisms of lncRNAs in breast cancer are still not fully understood.

In this study, we investigated the expression and potential role of the lncRNA SNORD3A in breast cancer. We confirmed the downregulation of SNORD3A in breast cancer cells and tissues and demonstrated a novel mechanism by which SNORD3A regulates chemosensitivity to 5 -fluorouracil (5-FU) in breast cancer.

\section{Materials and methods}

\section{Cell culture, transfection and tissue samples}

MCF10A, MCF-7, MDA-MB-231, T47D, SKBR3, ZR7530, BT549, HCC1937, BT474, and HEK293T cell lines were obtained from ATCC (Rockville, MD, USA) and cultured under standard conditions in media containing $10 \%$ fetal bovine serum (Gibco, Carlsbad, CA, USA).

To establish stable transfectants, cell lines were transfected with pReceiver-Lv201 lentiviral vectors containing SNORD3A or miR-185-5p, EX-T1651-Lv217 lentiviral vector containing Meis1, and psi-LVRU6GP vector with
UMPS short hairpin RNAs (target sequence for sh-1\#: 5'CCAAUCAAAUUCCAAUGCU-3', sh-2\#: 5'-GAGUUGAUAACUCUGGCAA-3') using Lipofectamine 3000 (Invitrogen, Carlsbad, CA, USA) following the manufacturer's instructions.

To inhibit miR-185-5p function, cells were transfected with miR-185-5p inhibitor (miRCURY LNA ${ }^{\mathrm{TM}}$ microRNA inhibitor for miR-185-5p; Exiqon, Vedbaek, Denmark).

Frozen fresh and paraffin-embedded breast cancer and non-cancerous tissues were collected from patients at the Affiliated Cancer Hospital of Guangzhou Medical University. All samples were collected with informed consent from patients, and all procedures were performed after the internal review and approval of the Ethics Committees of Guangzhou Medical University and the Affiliated Cancer Hospital.

\section{RNA immunoprecipitation (RIP) assay}

HEK293T cells were co-transfected with various cloned MS2bs vectors (MS2bs, MS2bs-SNORD3A-WT or MS2bs-SNORD3A-Mut) and MS2bp-GFP overexpression vector (Addgene, Watertown, MA, USA). After 48 h, RIP was performed with the EZ-Magna RIP Kit (Millipore, Burlington, MA, USA) using anti-green fluorescent protein (anti-GFP) according to the manufacturer's instructions. After RNA extraction, miR-185-5p level was examined by quantitative reverse transcriptase polymerase chain reaction (qRT-PCR).

\section{Xenograft model in athymic mice}

Female BALB/c athymic nude mice were obtained from Guangdong Medical Laboratory Animal Center, China. Cell lines were injected subcutaneously into the armpit of female BALB/c athymic nude mice to generate xenograft tumors ( $n=5 /$ group). Ten days after cancer cell implantation, mice were injected intraperitoneally with 5-FU $(30 \mathrm{mg} / \mathrm{kg}$ ) every 3 days for six cycles. At the experimental endpoint, animals were sacrificed, and then tumors were harvested and weighed. The animal studies were approved by the Institutional Animal Care and Use Committee (IACUC) of Guangzhou Medical University. Standard animal care and laboratory guidelines were conducted according to the IACUC protocol.

qRT-PCR, proliferation assay, colony-formation assay, chemosensitivity assay, western blot, luciferase reporter assay, chromatin immunoprecipitation (ChIP)-qPCR assay, in situ hybridization (ISH), immunohistochemistry (IHC) methods and primers are described in Supplemental Experimental Procedures.

\section{Statistical analysis}

Data are presented as mean \pm s.d. Student's $t$ test and $\chi^{2}$ test were used to compare the differences among different groups and correlation analysis. Survival curves were plotted 
using the Kaplan-Meier method and compared using logrank test. Statistical analyses were performed using GraphPad Prism 6. $p<0.05$ was considered statistically significant.

\section{Results}

LncRNA SNORD3A is downregulated in breast cancer

We first examined the expression level of the annotated potential lncRNA SNORD3A in a series of breast cancer cell lines and the normal mammary epithelial cell line MCF10A. The results demonstrated that SNORD3A expression was downregulated in breast cancer cells compared with MCF10A cells (Fig. 1a). We then examined the expression pattern of SNORD $3 A$ in paired breast cancer tissues and adjacent non-cancerous tissues. SNORD3A expression was also downregulated in breast cancer tissues compared with levels in paired noncancerous mammary tissues (Fig. 1b).

SNORD3A is a small nucleolar RNA located on human chromosome 17. SNORD3A is composed of three exons, with a transcript length of $699 \mathrm{nt}$. We analyzed the SNORD3A transcript using the online software (RegRNA 2.0, LNCipedia 5.0, Coding Potential Calculator), which predicted no protein-coding potentiality of SNORD3A (Fig. 1c). CPAT online software analysis also predicted no protein-coding ability but predicted an open reading frame (ORF) in the SNORD3A transcript (Fig. 1c).

To determine whether the SNORD3A ORF has coding potentiality, we constructed a series of vectors with a mutation in the initiation codon of GFP (the start codon ATGGTG was mutated to ATTGTT) that was fused downstream of the full SNORD3A transcript or potential ORF (Fig. 1d). While GFP expression was detected in HEK293T cells transfected with the wild-type GFP vector, no substantial expression of GFP was observed in HEK293T cells transfected with full-length-GFPmut or ORF-GFPmut construct (Fig. 1d). Western blot analysis using the anti-GFP antibody further confirmed that SNORD3A lacks protein-coding ability (Fig. 1e). We also examined the subcellular location of SNORD3A and found that $S N O R D 3 A$ resides in both the nucleus and the cytoplasm of breast cancer cells (Fig. 1f). These data indicated that SNORD3A as an IncRNA is downregulated in breast cancer cells and tissues.

\section{SNORD3A specifically enhances the chemosensitivity of breast cancer cells to 5 -FU}

To explore the biological function of $S N O R D 3 A$ in breast cancer, SNORD3A was stably overexpressed in MCF-7 and MDA-MB-231 cells (Fig. S1a). Cell proliferation assays showed that SNORD3A overexpression had no significant effect on proliferation (Fig. S1b). Colony-formation assay also showed that SNORD $3 \mathrm{~A}$ overexpression had no significant effect on colonyformation capacity of breast cancer cells (Fig. S1c).
We then examined whether SNORD3A was involved in the chemosensitivity of breast cancer cells. SNORD3Aoverexpressing MCF-7 and MDA-MB-231 cells and controls were treated with 5-FU, cisplatin (cDDP), or paclitaxel (PTX) at different concentrations. We found that $S N O R D 3 A$ overexpression specifically enhanced the chemosensitivity of breast cancer cells to 5 -FU but not to cDDP and PTX (Fig. 2a and Fig. S2a). Both plate colonyformation and soft agar colony-formation assays also confirmed that SNORD3A overexpression specifically promoted the chemosensitivity of breast cancer cells to 5FU (Fig. 2b, c and Fig. S2b, c).

The 5-FU antimetabolite drug has been widely used for the treatment of different types of cancer. To investigate whether SNORD3A enhanced the chemosensitivity of breast cancer cells to 5 -FU via modulating a metabolic pathway for 5-FU, we examined the expression of 5-FU metabolic-related genes, including uridine monophosphate synthetase (UMPS), thymidylate synthase (TS), dihydropyrimidine dehydrogenase (DPD), and methylene tetrahydrofolate reductase (MTHFR). SNORD3A overexpression had no effect on the expression of TS, DPD, and MTHFR in MCF7 and MDA-MB-231 cells, whereas SNORD3A overexpression markedly increased the UMPS protein level but not the mRNA level (Fig. $2 \mathrm{~d}$ and Fig. S2d). To determine whether SNORD3A enhancement of the sensitivity to 5 -FU in breast cancer cells is dependent on UMPS, we stably knocked down UMPS in breast cancer cells with ectopic SNORD3A overexpression (Fig. 2e). Knockdown of UMPS significantly diminished the promoting effect of SNORD3A overexpression on 5-FU sensitivity (Fig. 2f-h and Fig. S3a-c). Together, these data suggest that $S N O R D 3 A$ may promote 5 -FU sensitivity by activating UMPS expression in breast cancer cells.

\section{SNORD3A promotes UMPS expression via interacting with miR-185-5p}

Our results demonstrated that $S N O R D 3 A$ overexpression increased UMPS protein level but did not alter UMPS mRNA level in breast cancer cells. We next investigated the mechanism by which SNORD3A regulates UMPS. Recent studies showed that lncRNAs can function as competing endogenous RNAs (ceRNAs) by acting as molecular sponges for microRNAs (miRNAs), thus regulating gene expression at the posttranscriptional level. We used the online DIANA tool (http://diana.imis.athena-innovation.gr) and TargetScan (http://www.targetscan.org) to screen potential miRNAs that may bind with the SNORD3A transcript and the 3'-untranslated region (UTR) of UMPS mRNA. We found that miR-185-5p may bind with SNORD3A transcript and UMPS mRNA 3'-UTR (Fig. 3a). The interaction between SNORD3A and miR-185-5p was further predicted, and the minimum free energy at the binding site was calculated using RNAhybrid (Fig. 3b). 


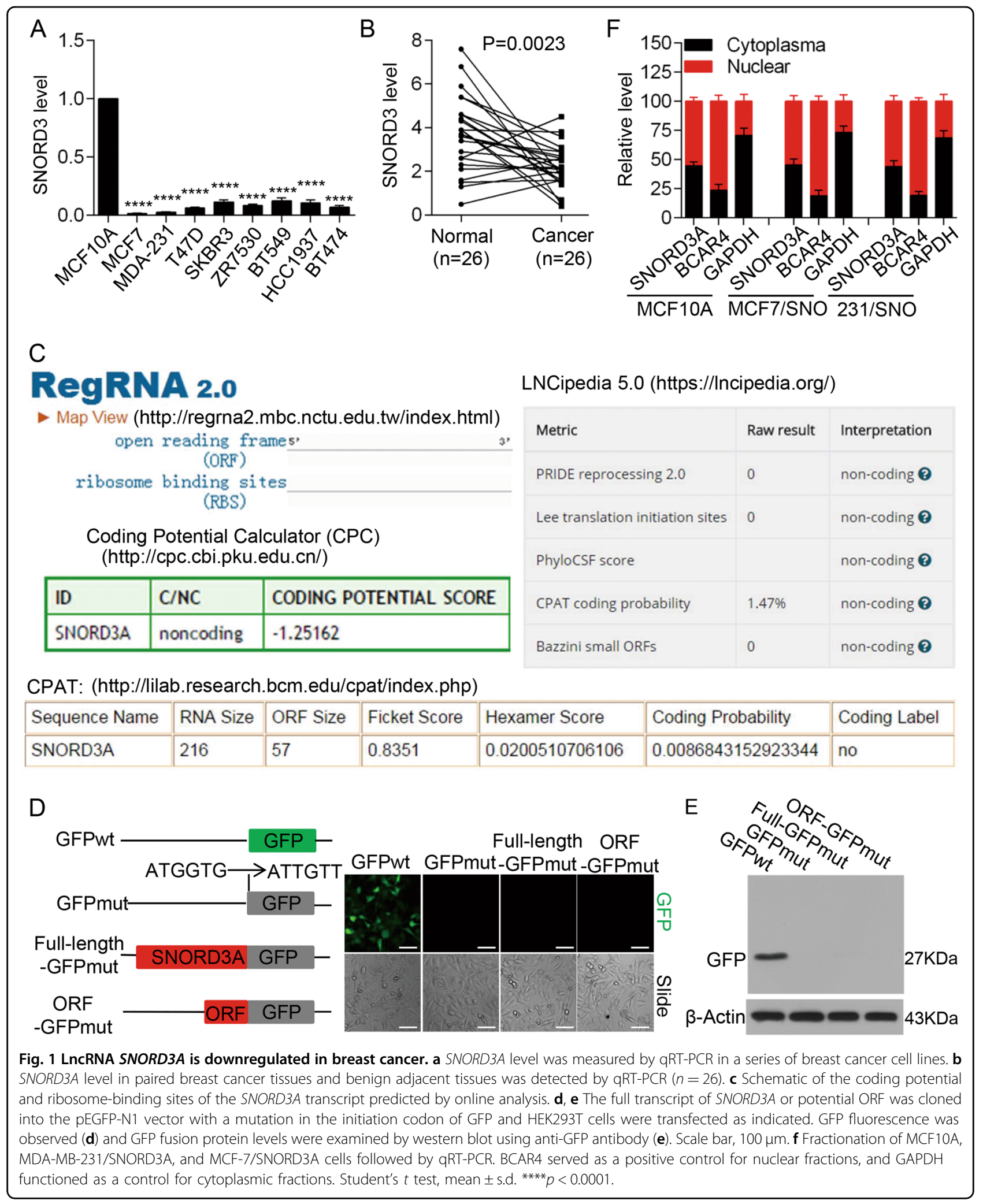

To determine whether SNORD3A interacts with miR$185-5 \mathrm{p}$ via the predicted binding sites, wild-type $S N O R D 3 A$ or SNORD3A with deletion mutations in the
miR-185-5p targeting site were cloned into the MS2b plasmid to transcribe RNA combined with MS2-binding sequences and then co-transfected with the MS2bp-GFP 


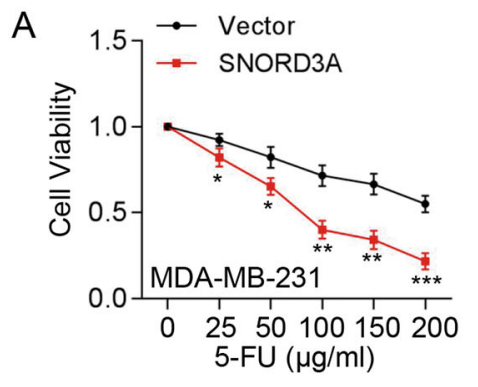

B
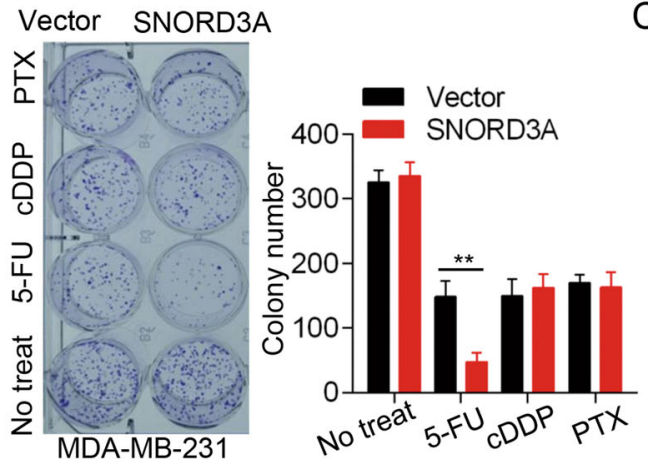

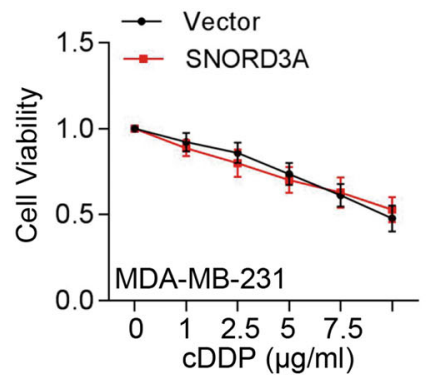

C Vector SNORD3A
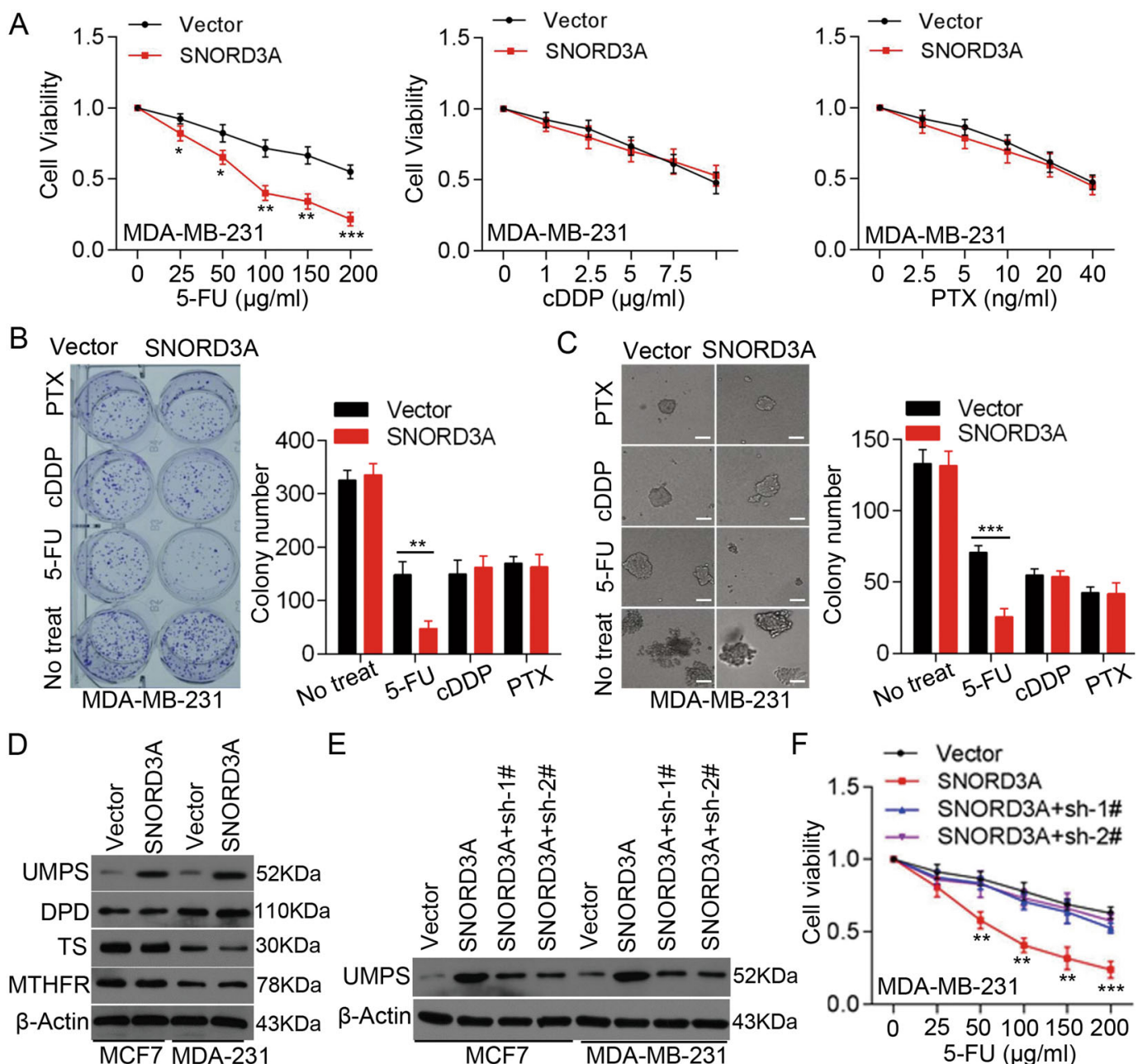

E

$\mathrm{F}$

$$
\text { notreat 5-FU CDDP PTX }
$$
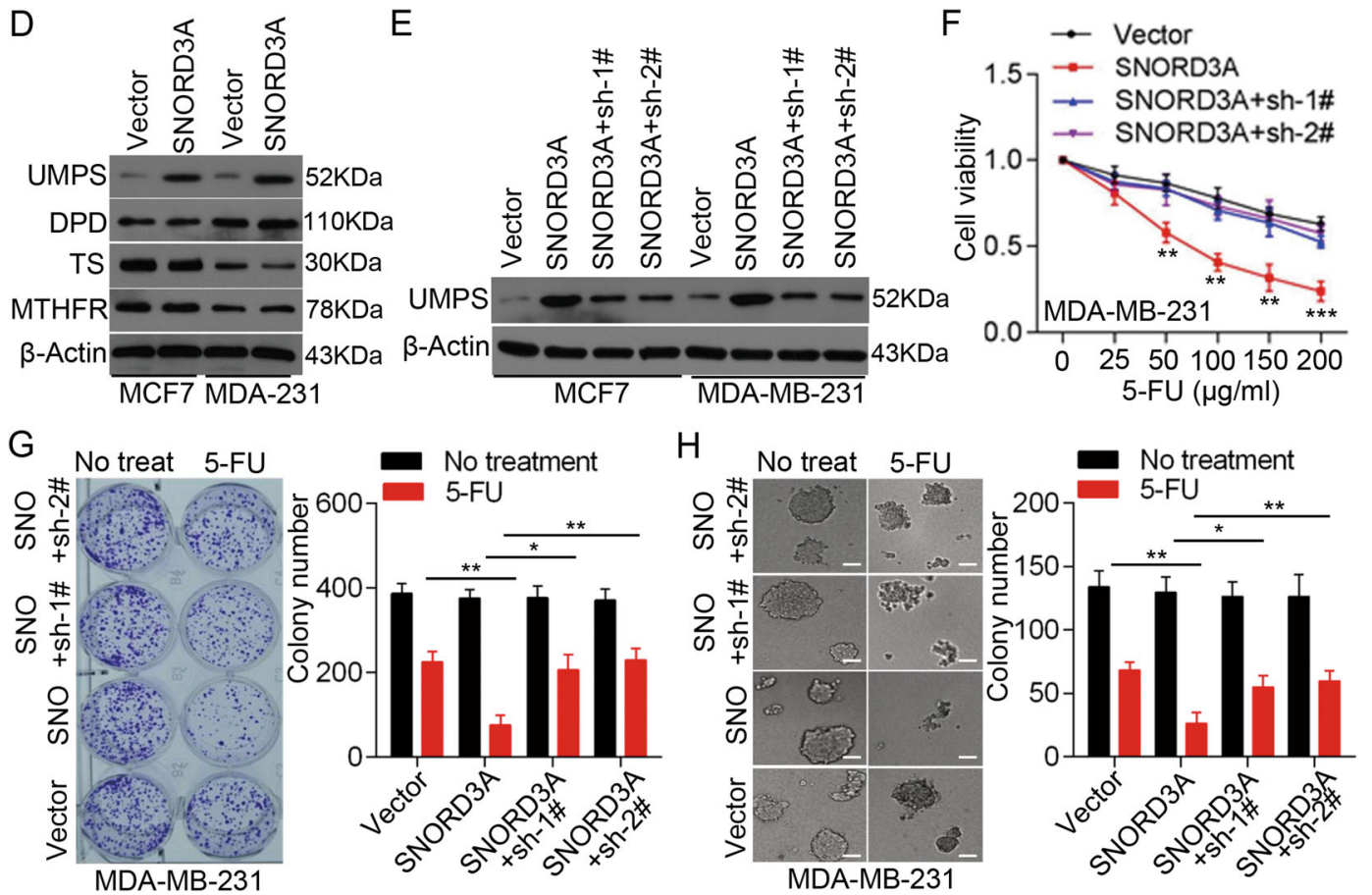

Fig. 2 SNORD3A specifically enhances the chemosensitivity of breast cancer cells to 5-FU. a The effects of SNORD3A overexpression on the chemosensitivity of MDA-MB-231 cell to 5-FU, cisplatin (CDDP), and paclitaxel (PTX) were detected by MTS assay at $72 \mathrm{~h}$ post-treatment. $\mathbf{b}$, $\mathbf{c}$ Colonyformation assay $(\mathbf{b})$ and soft agar colony-formation assay $(\mathbf{c})$ were performed to evaluate the colony growth of MDA-MB-231 cells under treatment with 5-FU $(100 \mu \mathrm{g} / \mathrm{ml})$, cDDP $(5 \mu \mathrm{g} / \mathrm{ml})$, or PTX $(10 \mathrm{ng} / \mathrm{ml})$. Scale bar, $100 \mu \mathrm{m}$. d Western blot showing UMPS, DPD, TS, and MTHFR protein levels in MCF-7 and MDA-MB-231 cells with or without SNORD3A overexpression. e UMPS protein levels in MCF-7 and MDA-MB-231 cells with SNORD3A overexpression and UMPS knockdown were examined by western blot. $\mathbf{f}$ Knockdown of UMPS abrogated the effects of SNORD3A overexpression on the chemosensitivity of MDA-MB-231 cells to 5 -FU by MTS assay at $72 \mathrm{~h}$ post-treatment. $\mathbf{g}, \mathbf{h}$ Colony growth of MDA-MB-231 cells with simultaneous expression interference of SNORD3A and UMPS were detected by colony-formation assay ( $\mathbf{g}$ ) and soft agar colony-formation assay (h). Scale bar, $100 \mu \mathrm{m}$. Student's $t$ test, mean \pm s.d. ${ }^{*} p<0.05,{ }^{* *} p<0.01,{ }^{* * *} p<0.001$. 


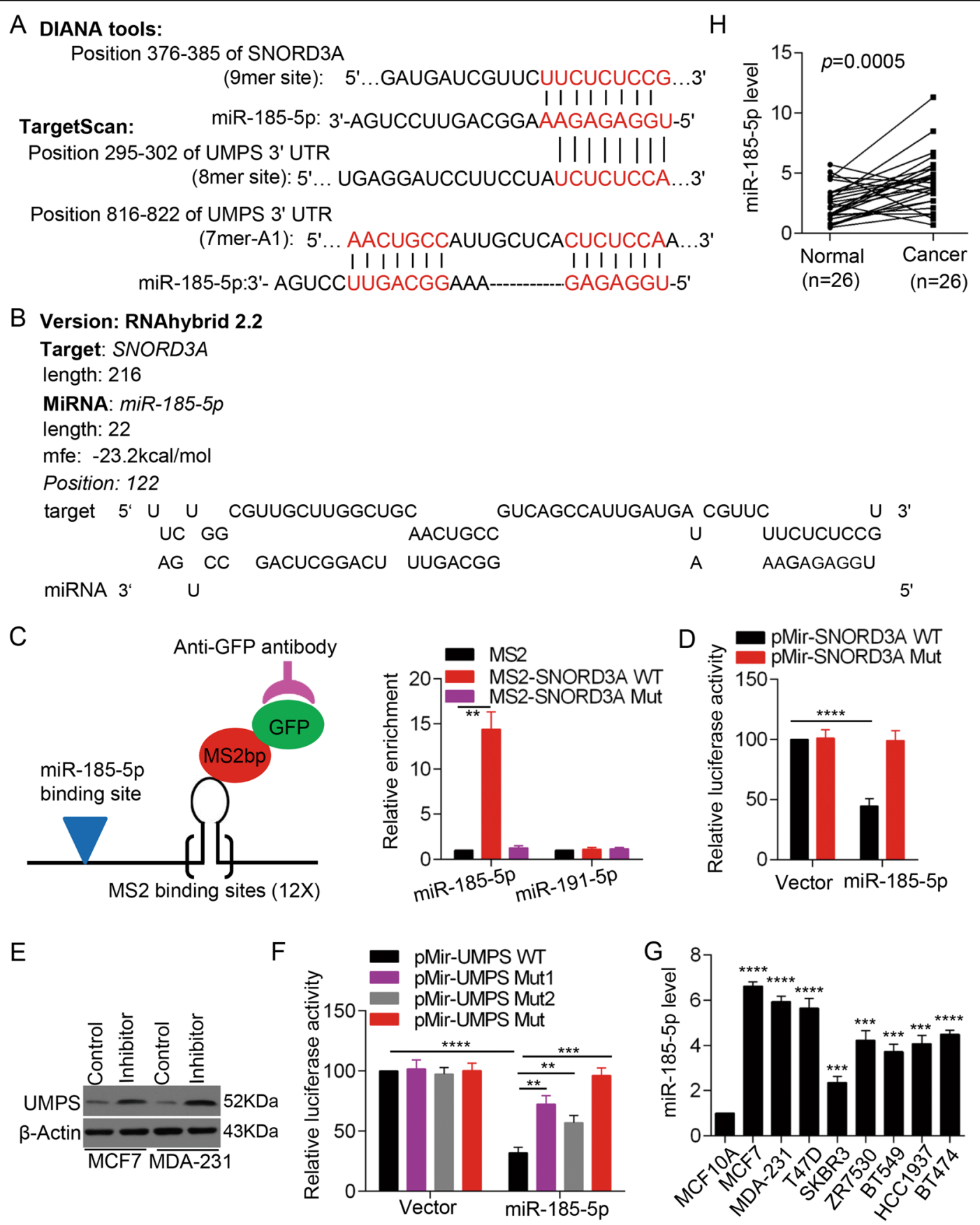

Fig. 3 SNORD3A promotes UMPS expression via interacting with miR-185-5p. a Schematic illustration of the interaction between SNORD3A and miR-185-5p predicted with DIANA tools (http://carolina.imis.athena-innovation.gr/diana_tools/web/index.php) and between miR-185-5p and UMPS mRNA 3'-UTR predicted by TargetScan (http://www.targetscan.org/vert_72/). b The interaction between SNORD3A and miR-185-5p and the minimum free energy (mfe) were predicted with the RNAhybrid software (https://bibiserv.cebitec.uni-bielefeld.de/rnahybrid). c MS2-RIP assay followed by qRT-PCR to detect the association between SNORD3A and miR-185-5p. miR-191-5p served as a negative control. $\mathbf{d}$ Luciferase activity demonstrated that miR-185-5p targeted SNORD3A. e Western blot was performed to detect UMPS protein levels in MCF-7 and MDA-MB-231 cells after transfection with miR-185-5p inhibitor. $\mathbf{f}$ Luciferase activity indicated that miR-185-5p targeted UMPS. $\mathbf{g}$ miR-185-5p expression levels in human breast cancer cell lines were detected by qRT-PCR. $\mathbf{h}$ miR-185-5p levels in breast cancer tumor tissues and paired non-tumorous mammary tissues were examined by qRT-PCR $(n=26)$. Student's $t$ test, mean \pm s.d. ${ }^{* *} p<0.01,{ }^{* * *} p<0.001,{ }^{* * * *} p<0.0001$. 
expression plasmid and miR-185-5p-expressing construct into HEK293T cells. We then performed RIP assays to pull-down miRNAs associated with SNORD3A via GFP antibody, followed by qRT-PCR. The results showed that miR-185-5p associated with SNORD3A via the targeting site (Fig. 3c). In contrast, miR-191-5p, a negative control, did not associate with SNORD3A. To further confirm the interaction of SNORD3A with miR-185-5p, we constructed luciferase reporters containing wild-type SNORD3A or SNORD3A with deletion mutation of the miR-185-5p targeting site. Luciferase constructs were cotransfected with miR-185-5p plasmid into HEK293T cells. We found that miR-185-5p reduced the reporter activity of the construct with wild-type SNORD3A (Fig. 3d).

We next examined whether UMPS is a direct target of miR-185-5p. miR-185-5p inhibitor upregulated UMPS protein levels in MCF-7 and MDA-MB-231 cells (Fig. 3e). We also constructed luciferase reporters containing the wild-type 3'-UTR UMPS mRNA or construct with mutated miR-185-5p targeting sites. miR-185-5p reduced the reporter activity of the construct with the wild-type 3'UTR of UMPS mRNA (Fig. 3f), suggesting that miR-185$5 p$ negatively regulates UMPS mRNA.

We further found that miR-185-5p expression was upregulated in breast cancer cell lines compared with the normal mammary epithelial cell line MCF10A (Fig. 3g). We also determined the expression of miR-185-5p in the cohort of the paired breast cancer and benign adjacent tissues and found that miR-185-5p levels were higher in breast cancer tissues than that in the paired noncancerous mammary tissues (Fig. 3h). Collectively, these data suggested that miR-185-5p is highly expressed in breast cancer and that SNORD3A acts as a sponge to miR185-5p to promote UMPS expression in breast cancer cells.

miR-185-5p is involved in SNORD3A-mediated sensitization to 5 -FU in breast cancer cells

Given that SNORD3A enhanced chemosensitivity to 5FU depending on UMPS and that SNORD3A acts as miR185-5p sponge to promote UMPS expression in breast cancer cells, we examined the involvement of miR-185-5p in SNORD3A-mediated chemosensitization to 5-FU. miR185-5p was stably overexpressed in MDA-MB-231 and MCF-7 cells with SNORD3A overexpression. MTS assays demonstrated that miR-185-5p overexpression diminished SNORD3A-induced sensitization to 5-FU (Fig. 4a and Fig. S4a). Moreover, both plate colony-formation and soft agar colony-formation assays confirmed that miR185-5p overexpression reversed SNORD3A-induced sensitization to 5-FU (Fig. 4b, c and Fig. S4b, c).

We further evaluated the biological role of SNORD3A and miR-185-5p in vivo. We subcutaneously injected MDA-MB-231 cells with SNORD3A overexpression combined with or without miR-185-5p overexpression or control MDA-MB-231 cells into nude mice. Mice were then treated with 5-FU as described in "Methods." SNORD3A overexpression significantly enhanced chemosensitivity to 5-FU compared with control mice treated with 5-FU. The volume and weight of tumors derived from SNORD3A-overexpressing cells were significantly decreased compared with those of control xenografts in response to 5-FU (Fig. 4d). However, miR-185-5p overexpression disrupted the effect of SNORD3A overexpression on chemosensitivity to 5-FU (Fig. 4d). Together these in vitro and in vivo results indicated that miR-185-5p is involved in SNORD3A-mediated sensitization to 5 -FU in breast cancer cells.

\section{SNORD3A expression is regulated by Meis 1 in breast cancer cells}

Given that SNORD3A expression was downregulated in breast cancer cells and tissues, we next explored the mechanisms underlying SNORD3A downregulation. To identify the transcription factors that regulate $S N O R D 3 A$ transcription, we used the JASPAR database (http://jaspar. binf.ku.dk) to predict potential transcription factorbinding sites in the 2-kb region upstream of the transcription start site in the SNORD3A gene. The analysis identified four potential Meis1-binding sites in the SNORD3A promoter (Fig. 5a). We further found that Meis1 expression was downregulated in breast cancer cells compared with the normal mammary cell line MCF10A (Fig. 5b). Meis1 level was also decreased in breast cancer tissues from the cohort compared with noncancerous tissues (Fig. 5c). Correlation analysis indicated that Meis1 level was positively correlated with SNORD3A level in breast cancer tissues (Fig. 5d). To investigate the potential role of Meis1 in regulating SNORD3A expression, Meis1 was overexpressed in MCF-7 and MDA-MB231 cell lines. Meis1 overexpression increased SNORD3A expression (Fig. 5e) as well as UMPS, the downstream target of SNORD3A (Fig. 5f). We next performed ChIP assays to confirm Meis1 occupancy on the SNORD3A promoter and found that Meis1 overexpression resulted in enrichment of Meis1 at the predicted Site C and Site D in the SNORD3A promoter in MCF-7 and MDA-MB-231 cells (Fig. 5g). Together these results suggested that Meis1 transcriptionally regulates $S N O R D 3 A$ expression in breast cancer cells.

\section{Clinical significance of SNORD3A-related signaling in breast cancer patients}

To further define the role of $S N O R D 3 A$ and verify its correlation with the newly identified upstream regulator and downstream effectors in breast cancer clinical samples, we first examined SNORD3A and miR-185-5p levels via ISH in breast cancer tissues $(N=72)$. SNORD3A 


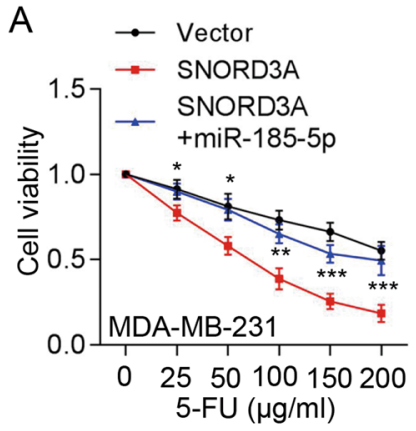

B

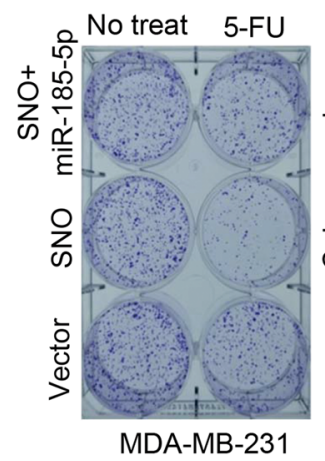

\section{$\mathrm{D}$}
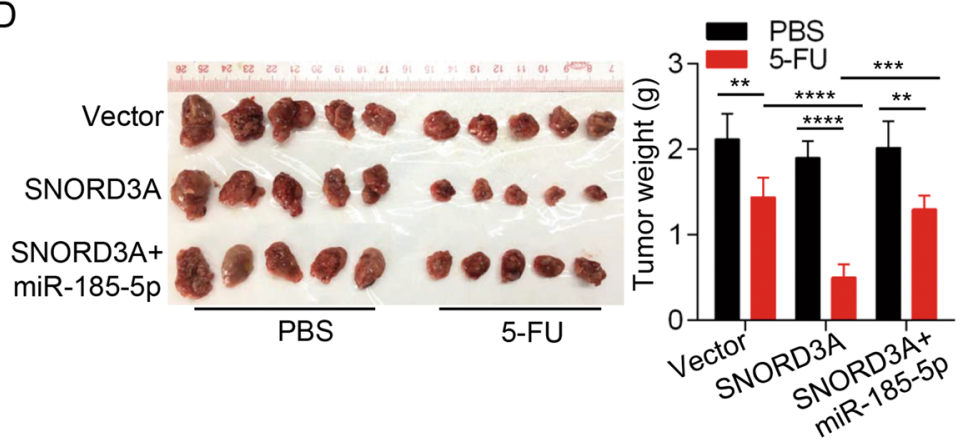

C
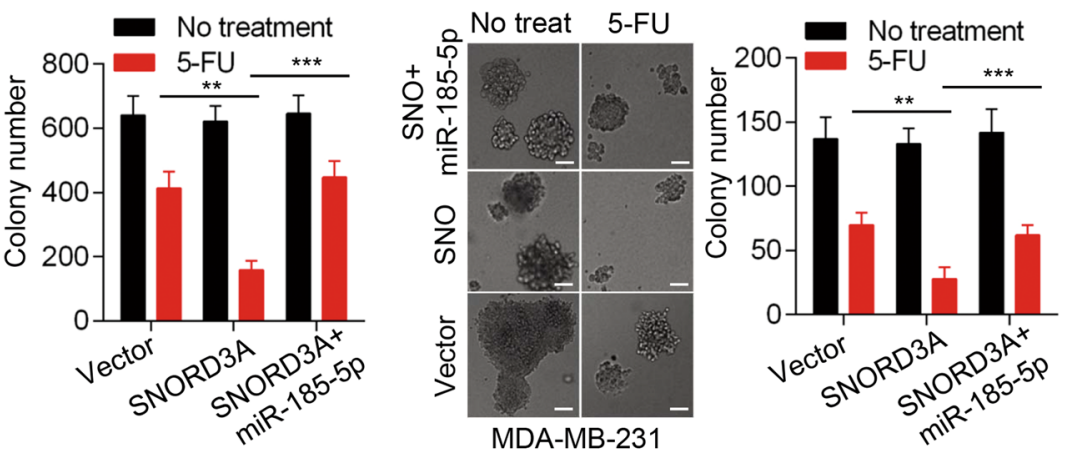

Fig. 4 miR-185-5p is involved in SNORD3A-mediated chemosensitization to 5-FU in breast cancer cells. a Ectopic expression of miR-185-5p diminished the effects of SNORD3A overexpression on the chemosensitization of MDA-MB-231 cells to 5-FU by MTS assay at $72 \mathrm{~h}$ post-treatment. $\mathbf{b}$, $\mathbf{c}$ Colony numbers of MDA-MB-231 cells with simultaneous expression interference of SNORD3A and miR-185-5p were detected by colony-formation assay (b) and soft agar colony-formation assay (c). Scale bar, $100 \mu \mathrm{m}$. d SNORD3A overexpression increased the chemosensitivity of MDA-MB-231derived tumors to 5-FU, but co-expressed miR-185-5p abrogated the effect of SNORD3A. $n=5 /$ group. Student's $t$ test, mean $\pm s . d .{ }^{*} p<0.05$, ${ }^{* *} p<$ $0.01,{ }^{* * *} p<0.001,{ }^{* * *} p<0.0001$.

transcript was expressed at low levels in approximately $68.06 \%$ of the specimens, while miR-185-5p transcript was highly expressed in approximately $73.61 \%$ of the specimens (Fig. 6a). We performed IHC staining of Meis1 and UMPS proteins in breast cancer tissues that were examined by ISH. As expected, Meis1 and UMPS protein were expressed at low levels in approximately $77.78 \%$ and 65.28\% breast cancer specimens, respectively (Fig. 6a). Further analysis showed that Meis1 level was positively correlated with SNORD3A level. UMPS level was positively correlated with SNORD3A level but negatively correlated with miR-185-5p level (Fig. 6b). Importantly, high SNORD3A transcript and Meis1 and UMPS protein levels predicts a better outcome, but high miR-185-5p level predicts a worse outcome in breast cancer patients receiving 5-FU-based chemotherapy (Fig. 6c).

We then used online tools to analyze the expression pattern and prognosis in breast cancer. Online analysis using UALCAN (http://ualcan.path.uab.edu) based on 1097 breast cancer tissues and 114 non-tumor mammary tissues indicated that Meis1 was downregulated in breast cancer tissues (Fig. 6d). We used a registration-free online service to generate Kaplan-Meier plots and found that high Meis1 expression in breast cancer predicted a better outcome in breast cancer (Fig. 6e), while high miR-185-5p predicted a worse outcome (Fig. 6f). There were no available records for SNORD3A in the online database. Together these data demonstrated the clinical significance of SNORD3A-mediated signaling in breast cancer patients.

\section{Discussion}

In this study, we for the first time delineated the expression pattern, critical role, and mechanisms of the lncRNA SNORD3A in breast cancer. Our findings provide several insights into the underlying mechanisms and potential strategy for enhancing 5-FU chemosensitivity in breast cancer: (1) SNORD3A as an IncRNA is downregulated in breast cancer (2) resulting from downregulation of the Meis1 transcription factor and (3) SNORD3A acts as a ceRNA of miR-185-5p (4) to upregulate UMPS protein expression and specifically enhance chemosensitivity to 5 -FU.

Emerging evidence has revealed the aberrant expression of IncRNAs in cancer and their critical roles in cancer development, progression, and $\operatorname{prognosis}^{18}$. Some 


A http://jaspar.binf.ku.dk/cgi-bin/jaspar_db.pl
\begin{tabular}{|l|l|l|l|l|l|l|l|l|}
\hline Model ID & Model name & Score & Relative score & Start & End & Strand & predicted site sequence \\
\hline MA0498.1 & Meis1 & 11.215 & 0.88354715355829 & 486 & 500 & 1 & gctgccactcacct & site A \\
MA0498.1 & Meis1 & 8.104 & 0.835861223641995 & 680 & 694 & 1 & agctgacacagaaaa & site B \\
MA0498.1 & Meis1 & 11.935 & 0.894583434348946 & 1984 & 1998 & 1 & agctgtcacatcatg & site C \\
MA0498.1 & Meis1 & 13.022 & 0.911245152709285 & 2758 & 2772 & 1 & ggctgtcattcagta & site D \\
\hline
\end{tabular}
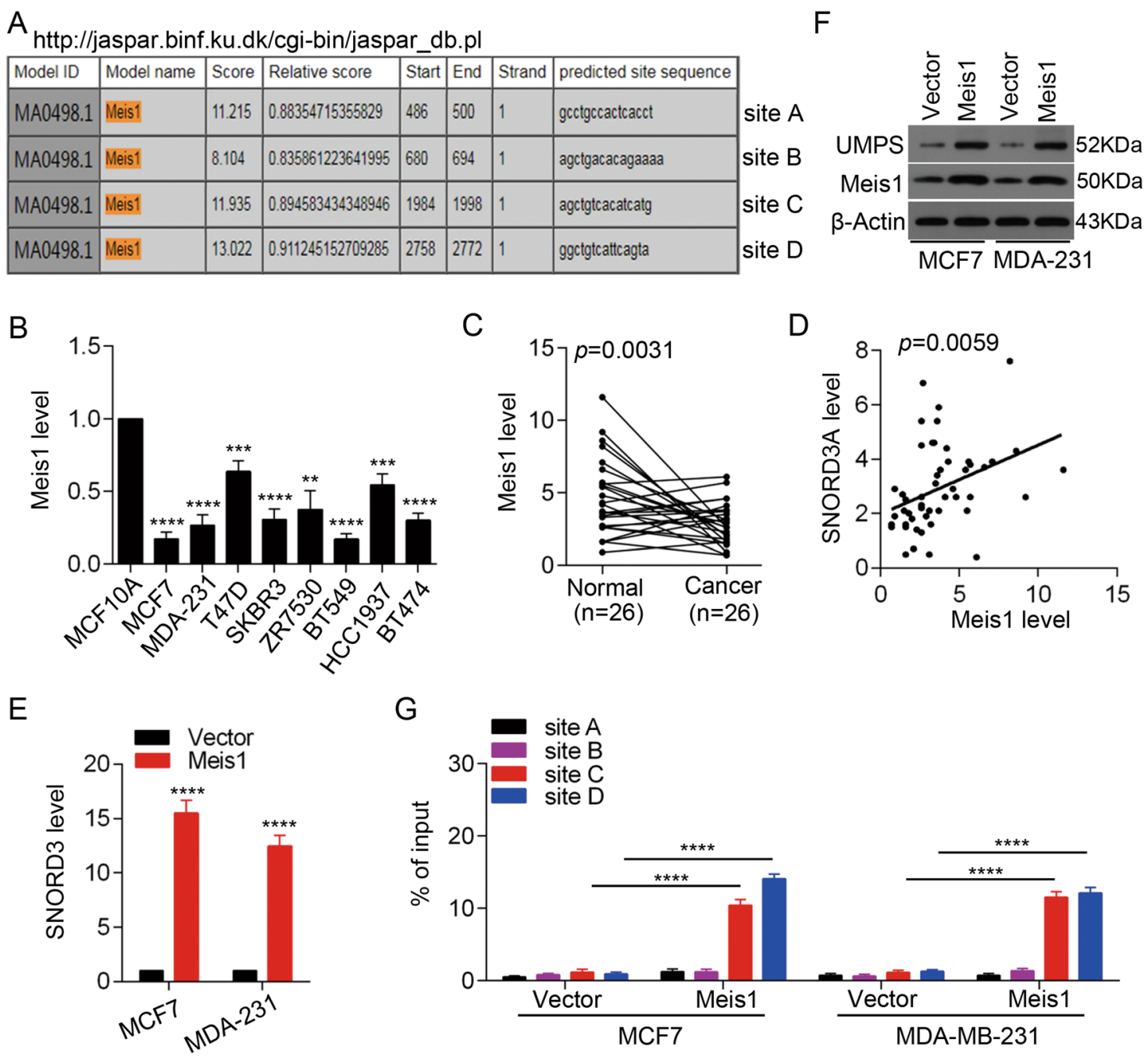

Fig. 5 SNORD3A expression is regulated by Meis1 in breast cancer cells. a Schematic illustration of the Meis1-binding sites in the potential SNORD3A promoter predicted by JASPAR database (http://jaspar.binf.ku.dk). b, c qRT-PCR was performed to detect the expression levels of Meis1 in breast cancer cell lines $(\mathbf{b})$ and in paired breast cancer tissues and normal mammary tissues $(n=26)(\mathbf{c})$. $\mathbf{d}$ The correlation between Meis1 and SNORD3A expression in breast cancer tissues was analyzed. e, $\mathbf{f}$ Meis 1 overexpression resulted in elevated mRNA level of SNORD3A (e) and protein level of UMPS in MCF-7 and MDA-MB-231 cells (f). $\mathbf{g}$ Enrichment of Meis1 protein on the SNORD3A promoter was determined by ChIP-qPCR assay. Student's $t$ test, mean \pm s.d. ${ }^{* *} p<0.001,{ }^{* *} p<0.001,{ }^{* * *} p<0.0001$.

IncRNAs have been reported to regulate important biological processes in cancer, such as proliferation ${ }^{19}$, metabolism $^{20}$, cancer stem cells $^{15}$, and metastasis ${ }^{21}$. LncRNAs deregulation has also been shown to be involved in the chemotherapeutic response and chemoresistance that is correlated with eventual cancer mortality ${ }^{22}$. Here we demonstrated low expression of lncRNA SNORD3A in breast cancer cells and tissues. SNORD3A overexpression had no significant effects on breast cancer cell proliferation and growth but specifically enhanced the chemosensitivity to 5-FU, validated by in vitro and in vivo studies. Importantly, higher SNORD3A level was positively correlated with clinical outcome in breast cancer patients receiving 5-FU-based chemotherapy. 5-FU is a pyrimidine analog that disrupts nucleoside metabolism and a widely used chemotherapy agent for some cancer types including breast cancer and colon cancer ${ }^{23} .5$ - FU is converted into its active metabolite fluorouridine monophosphate (FUMP), either directly by the orotate phosphoribosyltransferase domain of UMPS combining with phosphoribosyl pyrophosphate or indirectly via the activity of fluorouridine. FUMP is further phosphorylated and converted to fluorouridine triphosphate (FUTP). 5-FU induces cytotoxic effects and cell death through the incorporation of its active metabolite FUTP into RNA ${ }^{24}$. Abnormal alteration in the expression of 5-FU pathway genes directly correlates with chemosensitivity to $5-\mathrm{FU}^{25}$. Here we demonstrate that 


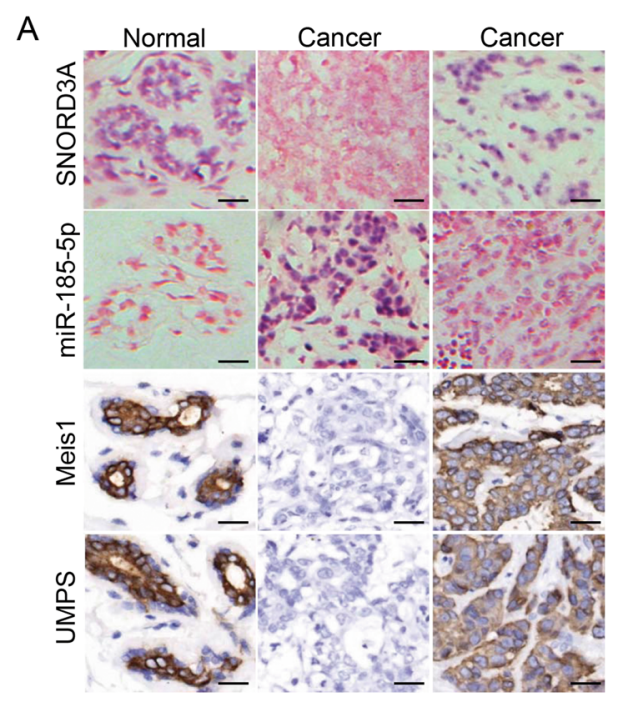

B

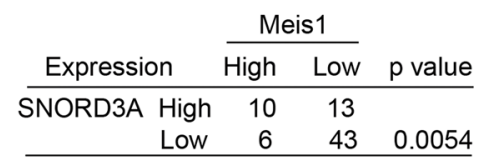

\begin{tabular}{ccccc} 
& & \multicolumn{2}{c}{ SNORD3A } & \\
\cline { 3 - 4 } \multicolumn{2}{c}{ Expression } & High & Low & p value \\
\hline UMPS & High & 14 & 11 & \\
& Low & 9 & 38 & 0.0029 \\
\hline
\end{tabular}

\begin{tabular}{ccccc} 
& & \multicolumn{2}{c}{ miR-185-5p } & \\
\cline { 3 - 4 } \multicolumn{2}{c}{ Expression } & High & Low & p value \\
\hline UMPS & High & 13 & 12 & \\
& Low & 40 & 7 & 0.0043 \\
\hline
\end{tabular}
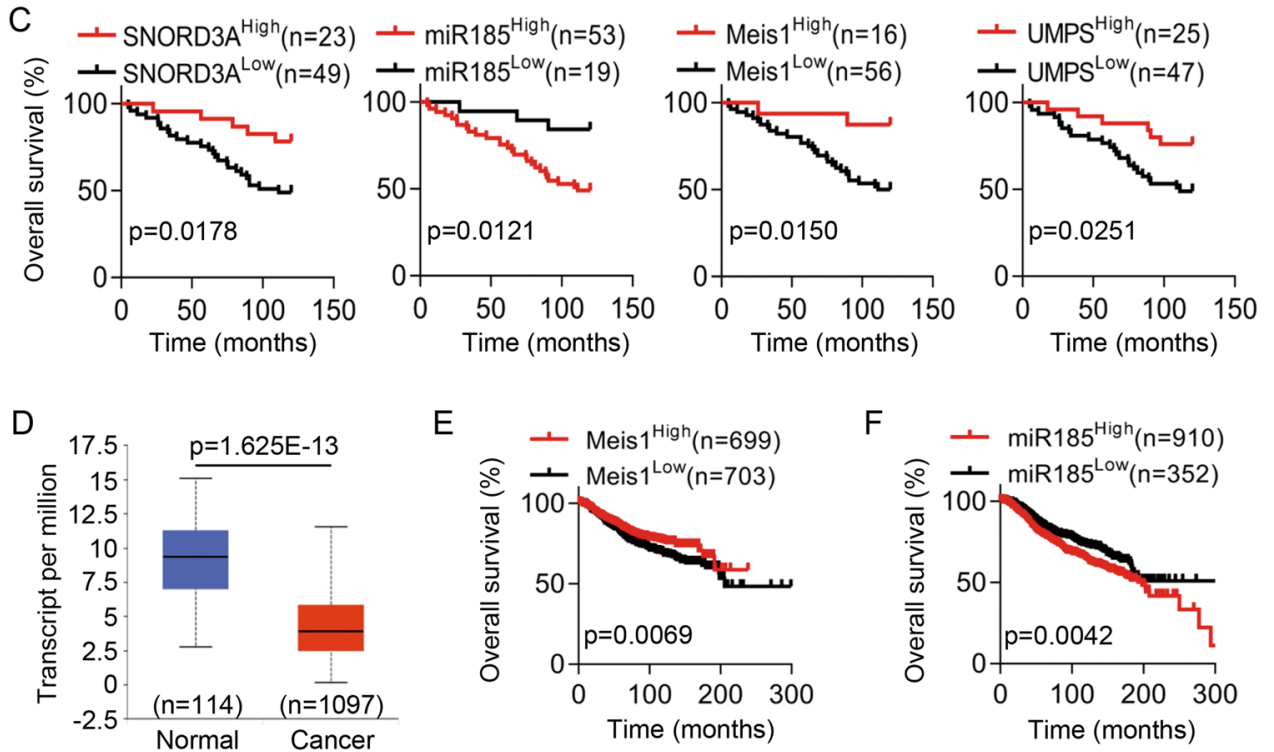

Fig. 6 Clinical significance of SNORD3A-related signaling in breast cancer patients. a SNORD3A transcript and miR-185-5p levels in breast cancer tissue samples were detected by in situ hybridization (ISH). Meis1 and UMPS protein levels in breast cancer tissue samples were detected by immunohistochemical staining (IHC) $(n=72)$. Scale bar, $50 \mu \mathrm{m}$. b Correlations between SNORD3A transcript level and Meis 1 protein level, between UMPS protein level and SNORD3A transcript level, and between UMPS protein level and miR-185-5p level in breast cancer tissues were analyzed. c Kaplan-Meier analysis indicated a correlation between low SNORD3A transcript level, Meis1 protein level, or UMPS protein level and poor overall survival in breast cancer patients and a correlation between high miR-185-5p level and poor overall survival in breast cancer patients. d UALCAN (http://ualcan.path.uab.edu) showed that Meis1 expression was downregulated in breast cancer tissues. e, f Kaplan-Meier plots according to Meis1 (e) and miR-185-5p (f) expression were generated for breast cancer patient cohorts in the TCGA database. Log-rank $p$ values are shown.

SNORD3A enhanced chemosensitization via inducing UMPS expression at the protein level and showed that UMPS knockdown impeded SNORD3A.

Given that SNORD3A increased UMPS expression at the protein level but not at the mRNA level, we next explored the molecular mechanism by which SNORD3A regulates UMPS expression. Some lncRNAs function as ceRNAs to regulate the functions of miRNAs through miRNA response elements ${ }^{26}$. The lncRNA H19 promotes
5-FU resistance in colorectal cancer (CRC) by sponging miR-194-5p and regulating SIRT1-mediated autophagy ${ }^{27}$. The lncRNA HOTAIR promotes gastric cancer progression by sponging miR-331-3p to upregulate HER2 expression $^{28}$. Exosome-transmitted lncARSR functions as a sponge of miR-34/miR-449 to induce c-MET and AXL expression to mediate sunitinib resistance in renal cell carcinoma $^{29}$. In this study, we found that SNORD3A shared miR-185-5p response elements with UMPS and 
facilitated UMPS expression by sponging miR-185-5p. miR-185-5p was highly expressed in breast cancer, which predicted a poor outcome in breast cancer patients. UMPS was experimentally validated to be a bona fide target of miR-185-5p. Ectopic miR-185-5p expression overcame the chemosensitization induced by SNORD3A in in vitro and in vivo models, indicating that SNORD3A acts as a ceRNA for miR-185-5p in breast cancer cells.

Recent studies reported the tumor-suppressive roles of miR-185-5p in cancer progression. miR-185 function is blocked by miR155HG, resulting in ANXA2 upregulation and glioblastoma growth and progression ${ }^{30}$. In CRC, miR185 downregulation by TCF1/LEF1 contributed to DCSIGN-induced cancer metastasis ${ }^{31}$. Interestingly, in nonsmall cell lung cancer, miR-185 inhibited cancer growth and invasion but increased chemosensitivity ${ }^{32}$. Here we showed that SNORD3A enhanced the sensitivity of breast cancer cells to 5-FU via sponging miR-185-5p and that miR-185-5p acts as a suppressive miRNA of UMPS to mediate the insensitivity to 5 -FU. Thus miR-185-5p may exhibit different effects in cancer progression and the therapeutic response. The precise effects of miR-185-5p and detailed mechanisms in cancer should be further validated by independent cohorts and prospective studies.

We also explored preliminary the mechanism for SNORD3A downregulation in breast cancer. Bioinformatics analysis combined with experimental validation indicated that Meis1 was downregulated in breast cancer and positively regulated SNORD3A expression at the transcriptional level. Clinically, low Meis1 level predicts a poor prognosis in breast cancer patients. Meis1, a 3amino acid loop extension class transcription factor, activates its target genes via interacting with Hox transcription factors ${ }^{33}$. Meis1 plays important roles in tumorigenesis, such as in the development of neuroblastomas $^{34}$, ovarian carcinomas $^{35}$, and leukemia ${ }^{36}$. Sebastian et al. reported that Meis1 enhances Syk signaling via downregulating miR-146a expression in Hoxa9driven acute myeloid leukemia ${ }^{37}$. The detailed molecular mechanisms for Meis1 transcriptionally regulating SNORD3A will be investigated in our next studies.

In conclusion, our study delineates the expression pattern, role, and mechanism of the lncRNA SNORD3A in breast cancer. SNORD3A is downregulated in breast cancer resulting from Meis1 downregulation. SNORD3A enhances UMPS expression by sponging miR-185-5p to specifically promote chemosensitivity to 5 -FU in breast cancer. Our findings provide insight into the SNORD3A-UMPS axis as a promising therapeutic target against breast cancer, with important translational implications for improving the efficacy of 5-FU for breast cancer patients. Further studies should be performed to develop precise strategies targeting SNORD3A-UMPS signaling.

\section{Acknowledgements}

This study was supported by grants from the National Natural Science Foundation of China (No.81672616 and No.81872197); grants from Guangdong Natural Science Funds for Distinguished Young Scholars (No.2016A030306003); Guangdong Special Support Program (No.2017TQ04R809); grants from Guangdong Natural Science Funds (2017A030313867), supported by Guangzhou key medical discipline construction project fund; Medical Scientific Research Foundation of Guangdong Province of China (No.B2018274); and grants from Science and Technology Program of Guangzhou, China (No.201710010100).

\section{Conflict of interest}

The authors declare that they have no conflict of interest.

\section{Publisher's note}

Springer Nature remains neutral with regard to jurisdictional claims in published maps and institutional affiliations.

Supplementary Information accompanies this paper at (https://doi.org/ 10.1038/s41419-020-2557-2).

Received: 8 January 2020 Revised: 20 April 2020 Accepted: 20 April 2020 Published online: 07 May 2020

\section{References}

1. Siegel, R. L., Miller, K. D. \& Jemal, A. Cancer statistics, 2017. CA Cancer J. Clin. 67 7-30 (2017).

2. Bray, F. et al. Global cancer statistics 2018: GLOBOCAN estimates of incidence and mortality worldwide for 36 cancers in 185 countries. CA Cancer J. Clin. $\mathbf{6 8}$, 394-424 (2018)

3. Perou, C. M. et al. Molecular portraits of human breast tumours. Nature 406, 747-752 (2000)

4. Prat, A. et al. Characterization of cell lines derived from breast cancers and normal mammary tissues for the study of the intrinsic molecular subtypes. Breast Cancer Res. Treat. 142, 237-255 (2013).

5. Shi, J. et al. Disrupting the interaction of BRD4 with diacetylated Twist suppresses tumorigenesis in basal-like breast cancer. Cancer Cell 25, 210-225 (2014).

6. Kartal-Yandim, M., Adan-Gokbulut, A. \& Baran, Y. Molecular mechanisms of drug resistance and its reversal in cancer. Crit. Rev. Biotechnol. 36, 716-726 (2016).

7. Lee, J. T. Epigenetic regulation by long noncoding RNAs. Science $\mathbf{3 3 8}$, 1435-1439 (2012)

8. Ulitsky, I. \& Bartel, D. P. lincRNAs: genomics, evolution, and mechanisms. Cell 154, 26-46 (2013).

9. Bartonicek, N., Maag, J. L. \& Dinger, M. E. Long noncoding RNAs in cancer: mechanisms of action and technological advancements. Mol. Cancer 15, 43 (2016).

10. Batista, P. J. \& Chang, H. Y. Long noncoding RNAs: cellular address codes in development and disease. Cell 152, 1298-1307 (2013).

11. Wang, K. C. \& Chang, H. Y. Molecular mechanisms of long noncoding RNAs. Mol. Cell 43, 904-914 (2011).

12. Luo, L. et al. LINC01638 InCRNA activates MTDH-Twist1 signaling by preventing SPOP-mediated c-Myc degradation in triple-negative breast cancer. Oncogene 37, 6166-6179 (2018).

13. Arab, K. et al. Long noncoding RNA TARID directs demethylation and activation of the tumor suppressor TCF21 via GADD45A. Mol. Cell 55, 604-614 (2014).

14. Yuan, J. H. et al. A long noncoding RNA activated by TGF-beta promotes the invasion-metastasis cascade in hepatocellular carcinoma. Cancer Cell 25, 666-681 (2014).

15. Wang, Y. et al. The long noncoding RNA IncTCF7 promotes self-renewal of human liver cancer stem cells through activation of Wnt signaling. Cell Stem Cell 16, 413-425 (2015).

16. Booy, E. P., McRae, E. K., Koul, A., Lin, F. \& McKenna, S. A. The long non-coding RNA BC200 (BCYRN1) is critical for cancer cell survival and proliferation. Mol. Cancer 16, 109 (2017). 
17. Liu, B. et al. A cytoplasmic NF-kappaB interacting long noncoding RNA blocks IkappaB phosphorylation and suppresses breast cancer metastasis. Cancer Cell 27, 370-381 (2015).

18. Bhan, A., Soleimani, M. \& Mandal, S. S. Long noncoding RNA and cancer: a new paradigm. Cancer Res 77, 3965-3981 (2017).

19. Liu, Y. W. et al. LincRNAFEZF1-AS1 represses p21 expression to promote gastric cancer proliferation through LSD1-Mediated H3K4me2 demethylation. Mol. Cancer 16, 39 (2017)

20. Bian, Z. et al. LnCRNA-FEZF1-AS1 promotes tumor proliferation and metastasis in colorectal cancer by regulating PKM2 signaling. Clin. Cancer Res. 24 4808-4819 (2018).

21. Gupta, R. A. et al. Long non-coding RNA HOTAIR reprograms chromatin state to promote cancer metastasis. Nature 464, 1071-1076 (2010).

22. Zeng F, et al. Linc00173 promotes chemoresistance and progression of small cell lung cancer by sponging miR-218 to regulate Etk expression. Oncogene 39, 293-307 (2020).

23. Kufe, D. W. \& Major, P. P. 5-Fluorouracil incorporation into human breast carcinoma RNA correlates with cytotoxicity. J. Biol. Chem. 256, 9802-9805 (1981).

24. Longley, D. B., Harkin, D. P. \& Johnston, P. G. 5-Fluorouracil: mechanisms of action and clinical strategies. Nat. Rev. Cancer 3, 330-338 (2003).

25. Kidd, E. A. et al. Variance in the expression of 5-fluorouracil pathway genes in colorectal cancer. Clin. Cancer Res. 11, 2612-2619 (2005).

26. Yoon, J. H., Abdelmohsen, K. \& Gorospe, M. Posttranscriptional gene regulation by long noncoding RNA. J. Mol. Biol. 425, 3723-3730 (2013).

27. Wang, M. et al. Long non-coding RNA H19 confers 5-Fu resistance in colorectal cancer by promoting SIRT1-mediated autophagy. Cell Death Dis. 9, 1149 (2018).
28. Liu, X. H. et al. LnC RNA HOTAIR functions as a competing endogenous RNA to regulate HER2 expression by sponging miR-331-3p in gastric cancer. Mol. Cancer 13, 92 (2014).

29. Qu, L. et al. Exosome-transmitted IncARSR promotes sunitinib resistance in renal cancer by acting as a competing endogenous RNA. Cancer Cell 29, 653-668 (2016)

30. Wu, W. et al. The miR155HG/miR-185/ANXA2 loop contributes to glioblastoma growth and progression. J. Exp. Clin. Cancer Res. 38, 133 (2019).

31. Yuan M, et al. DC-SIGN-LEF1/TCF1-miR-185 feedback loop promotes colorectal cancer invasion and metastasis. Cell Death Differ. 27, 379-395 (2020).

32. Zhou, C. W., Zhao, W. J., Zhu, Y. G. \& Zhao, X. D. MiR-185 inhibits tumor growth and enhances chemo-resistance via targeting SRY-related high mobility group box transcription factor 13 in non-small-cell carcinoma. Am. J. Transl. Res. 10, 2600-2609 (2018)

33. Moskow, J. J., Bullrich, F., Huebner, K., Daar, I. O. \& Buchberg, A. M. Meis1, a PBX1-related homeobox gene involved in myeloid leukemia in $\mathrm{BXH}-2$ mice. Mol. Cell. Biol. 15, 5434-5443 (1995).

34. Geerts, D., Schilderink, N., Jorritsma, G. \& Versteeg, R. The role of the MEIS homeobox genes in neuroblastoma. Cancer Lett. 197, 87-92 (2003).

35. Crijns, A. P. et al. MEIS and PBX homeobox proteins in ovarian cancer. Eur. J. Cancer 43, 2495-2505 (2007).

36. Argiropoulos, B., Yung, E. \& Humphries, R. K. Unraveling the crucial roles of Meis1 in leukemogenesis and normal hematopoiesis. Genes Dev. 21 2845-2849 (2007).

37. Mohr, S. et al. Hoxa9 and Meis1 cooperatively induce addiction to Syk signaling by suppressing miR-146a in acute myeloid leukemia. Cancer Cell 31, 549.e11-562.e11 (2017). 